\title{
Using experimental modal analysis to assess the behaviour of timber elements
}

\author{
Georges Kouroussis ${ }^{1, *}$, Lassaad Ben Fekih ${ }^{1}$, and Thierry Descamps ${ }^{2}$ \\ ${ }^{1}$ University of Mons, Faculty of Engineering, Department of the Theoretical Mechanics, Dynamics and Vibrations, Place du \\ Parc 20, 7000 Mons, Belgium \\ ${ }^{2}$ University of Mons, Faculty of Engineering, Department of Civil Engineering, Place du Parc 20, 7000 Mons, Belgium
}

Received: 12 December 2016 / Accepted: 6 November 2017

\begin{abstract}
Timber frameworks are one of the most important and widespread types of structures. Their configurations and joints are usually complex and require a high level of craftsmanship to assemble. In the field of restoration, a good understanding of the structural behaviour is necessary and is often based on assessment techniques dedicated to wood characterisation. This paper presents the use of experimental modal analysis for finite element updating. To do this, several timber beams in a free supported condition were analysed in order to extract their bending natural characteristics (frequency, damping and mode shapes). Corresponding ABAQUS finite element models were derived which included the effects of local defects (holes, cracks and wood nodes), moisture and structural decay. To achieve the modal updating, additional simulations were performed in order to study the sensitivity of the mechanical parameters. With the intent to estimate their mechanical properties, a procedure of modal updating was carried out in MatLab with a Python script. This was created to extract the modal information from the ABAQUS modal analysis results to be compared with the experimental results. The updating was based on a minimum of unconstrained multivariable function using a derivative-free method. The objective function was selected from the conventional comparison tools (absolute or relative frequency difference, and/or modal assurance criterion). This testing technique was used to determine the dynamic mechanical properties of timber beams, such as the anisotropic Young's Moduli and damping ratio. To verify the modulus, a series of static 4-point bending tests and STS04 classifications were conducted. The results also revealed that local defects have a negligible influence on natural frequencies. The results demonstrate that this assessment tool offers an effective method to obtain the mechanical properties of timber elements, especially when on-site and non-destructive techniques are needed, for example when retrofitting an existing structure.
\end{abstract}

Keywords: Structural behaviour assessment / modal analysis / timber beam / NDT / FRF measurement / modal updating

\section{Introduction}

Wood is widely used as main material in building construction due to its good thermal and acoustic properties, and to its aesthetic. Contrary to other building materials, wood is heterogeneous, anisotropic and hygroscopic. It is mainly composed of $50 \%$ carbon, $43 \%$ oxygen, $6 \%$ hydrogen and $1 \%$ nitrogen in mass, against the dry timber mass (all the carbon contained in the wood comes from the carbon dioxide used for photosynthesis). Beginning with the inside layer of a tree (Fig. 1), the wood is composed of:

\footnotetext{
* e-mail: georges.kouroussis@umons.ac.be
}

- Heartwood or duramen: This part is called "perfect wood" and only contains dead cells which ensure the role of support for the tree. It can be distinguished by its colour, as it is much darker than living wood.

- Sapwood constituted of living cells: This is the younger, outermost wood.

- Based generator or cambium: Located directly under the cork, the cambium produces the outside of the internal cork and the inside of the wood cells. In temperate zones, it works only in spring and summer and produces a layer of wood every year, called an annual growth ring, through which it is possible to distinguish the initial wood (spring) and the final wood (summer).

- Internal cork is the inner part of cork and conducts the sap containing sugars produced by photosynthesis. 


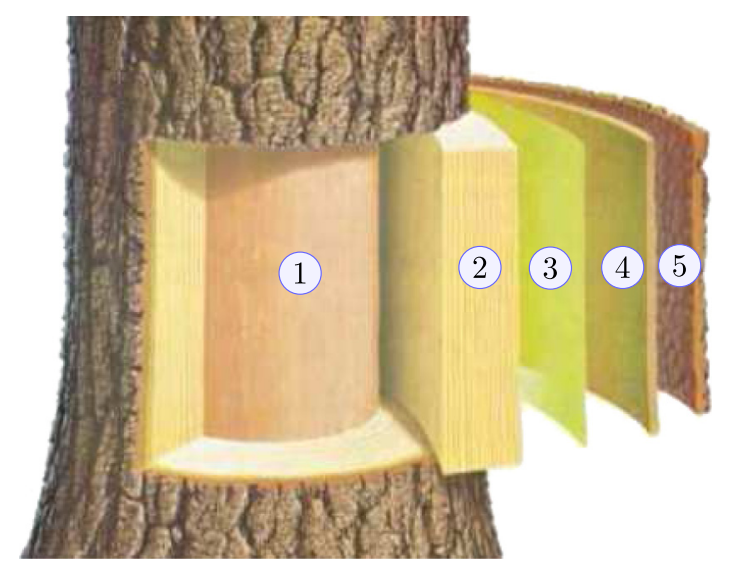

Fig. 1. Constitution of wood.

- External cork, more commonly called cork, which is composed of dead cells and acts as a protective coating.

Wood properties are affected by the climate and the conditions the tree's growth. Wood is also a porous material which can lose or regain moisture according to the relative moisture, and it can eventually store water for its cellular cavities. The variations of humidity influence a piece's dimensions and its mechanical strength. In general, the mechanical properties are considered for $12 \%$ of moisture content. As wood is a highly variable natural material, susceptible to physical, chemical and biological deterioration, it is very difficult to assess it from a mechanical point of view. Before any intervention on existing structures, the first step is to fully understand how the structure works, meaning that the knowledge of its mechanical properties is essential.

Sounding methods are the oldest methods used to inspect in situ timber members [1]. It consists in exciting the timber beams with a hammer. This requires a trained inspector able to interpret the sound. This method allows a quick screening of a timber member but is highly qualitative and not quantitative. The diagnosis may also change according to the inspector. The ultrasonic method offers a way to control the quality of timber product production [2]. It consists of measuring the time difference between the arrival of 2 piezo-electrical transducers in a frequency range of 20 $500 \mathrm{kHz}$ with an accuracy of $1 \mu \mathrm{s}$. The transducers are located on the two opposite sides of the timber piece. The data acquisition system consists of a signal detector, an analyser and a recorder which collects and retains the wave information. The measured propagation velocity depends on numerous factors, such as the species, moisture content, grain direction and density, and it allows the determination of Young's modulus as well as any possible defects in the wood. New generations of devices deal with the analysis of the acousto-ultrasonic response of the wood by measuring the speed of the transmitted wave and their maximal peak of energy. The speed of propagation is still correlated to Young's modulus but the energy is related to the local singularities, like knots or grain direction [3]. Other assessment techniques are available and their use must

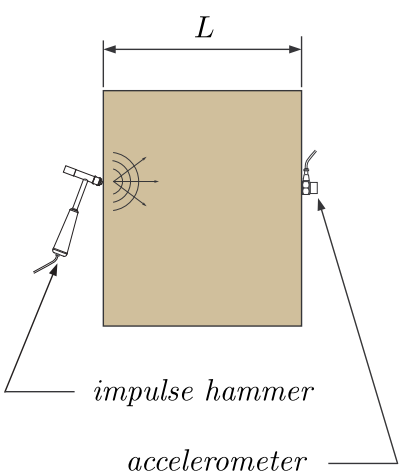

Fig. 2. Device generally used for the vibration method.

be discussed depending on the remaining level of doubt after visual inspection, the feasibility of repair/strengthening, and is always in combination with economical considerations.

Complementary to the existing non-destructive testing methods, the purpose of this work is to propose the first step in the development of an efficient technique for the mechanical assessment of old timber structures. The chosen method is the vibration technique, due to the simplicity of its implementation and the fact that it gives an overall view of the surveyed wood element. This paper presents the results investigated for the experimental assessment of the dynamic properties of timber elements, measured by forced vibration tests using modal identification methods.

\section{Existing vibration techniques for wood characteristics assessment}

The vibration method consists of exciting the structure by hitting it with a hammer at several points of impact in order to induce a stress wave into the wood specimen [1]. The acceleration signal is measured using small accelerometers (Fig. 2). When applied to wood inspection, the high attenuation of wood, which worsen with high frequencies, must be considered. However, high frequency transmissions are more sensitive to internal defects such as knots or other singularities. The choice of the frequency range results from a compromise between the level of attenuation (small or great spans) on the studied length $L$ and the sensitivity to defects. The frequency spectrum analysis can be used to assess and quantify timber decay. When fundamental modes are of interest, some formula exist to establish close relationships between Young's modulus and the studied fundamental mode.

The mechanical properties of a timber structure can be experimentally determined by forced or ambient vibration tests using dynamic properties or modal identification methods. These methods have been successfully used in several structural applications. Yang et al. [4] proposed to use the shape of the flexural vibration wave of wooden beams at the first mode as a primary indicator. The dynamic modulus of the elasticity of the studied beam sections was calculated from the ratio of the curvature of the corresponding mode shape. By using a cantilever beam 


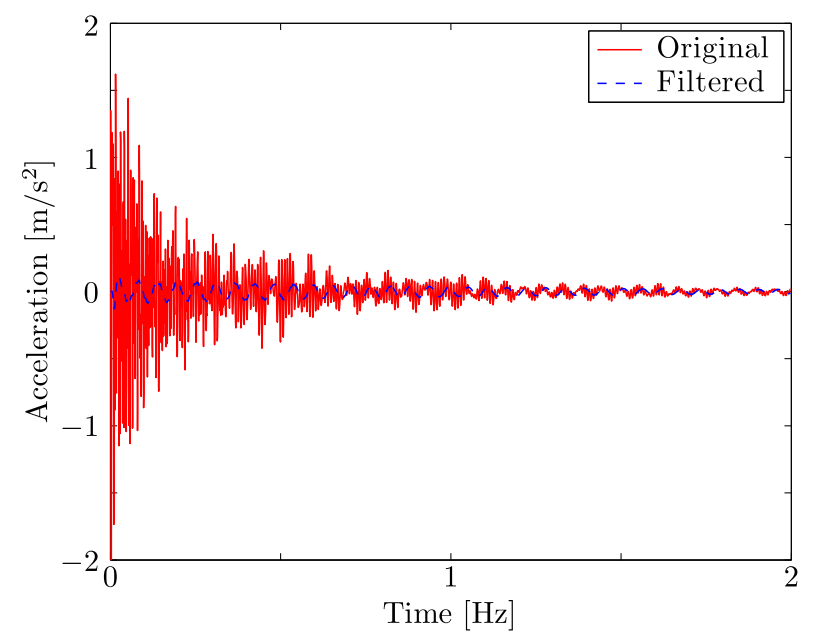

Fig. 3. Example of a measured acceleration on a cantilever wood beam due to an impact.

configuration, Wang et al. [5] detected the first bending mode of the beam, using a simple technique based on the measurement of impulse response. Both Young's modulus and the damping ratio were estimated. As illustrated in Figure 3, the main difficulty is to isolate the first bending mode among all the recorded bending modes (and eventually torsional modes if the symmetry is not verified). It is clearly shown that a filtering becomes unavoidable, with the lack of awareness of its cut-off frequency. Moreover, the proposed method needs to be performed on wood species under laboratory testing conditions. A similar method was used by Wang et al. [6] and dedicated to in situ measurement. An analytical model was derived from the flexural beam theory to correlate the associated eigenfrequency. An alternative non-destructive impact test, based on sectional mode shapes, was presented by Alves et al. [7]. This allows the determination of transverse Young's modulus of wood species.

From this short literature review, it is seen that existing vibration techniques are mainly focused on one flexural mode, and provide one or two dynamic parameters. The effect of each mechanical parameter on the natural frequency is also disregarded. Another finding is that one specific mode shape is expected to be analysed without verifying that the correct mode is analysed. As suggested by Olsson and Källsner [8], a calibration with a finite element model reduces the uncertainty in the mode estimation. However, the experimental data needs to be completely mastered by identifying the various mode shapes well.

\section{On the use of frequency response functions}

In mechanical engineering, model updating techniques are widely used in order to calibrate models in structural dynamics. The potential of model updating techniques has close links to inverse parameter identification, as long as the obtained values are inside a realistic range. In this paper, it will be shown how finite element updating with

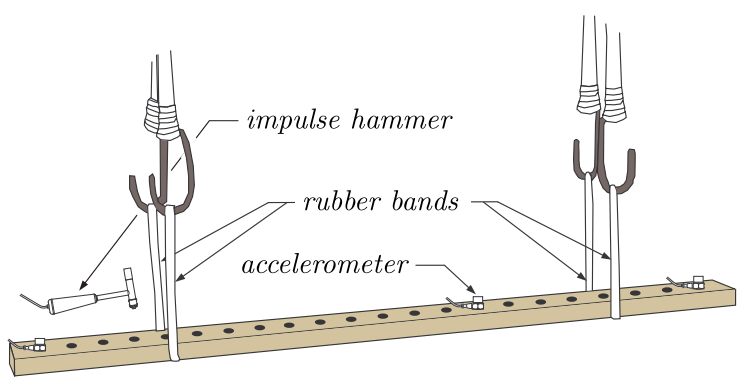

(a)

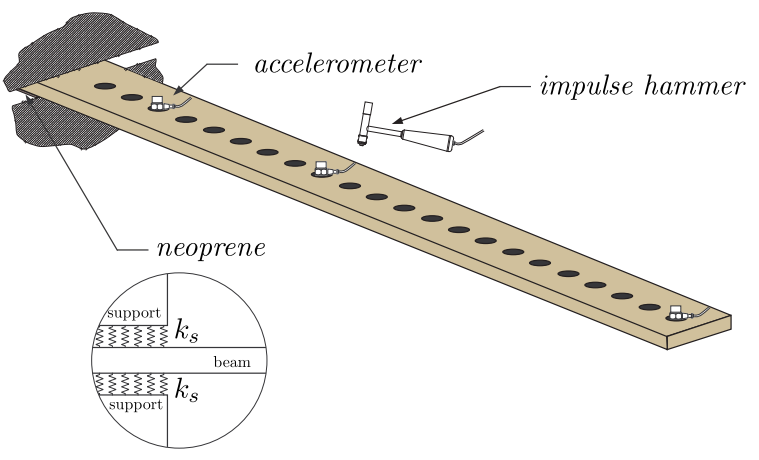

(b)

Fig. 4. Test support mechanism with hammer in (a) free-free conditions and (b) cantilever installation.

wood beams can be used. The feasibility of experimental modal analysis on the characterisation of timber element dynamic properties will also be described.

A series of beams (more than twenty specimens) of the same species (Douglas-fir) and dimensions $(2.4 \times 0.14$ $\times 0.04 \mathrm{~m})$ were analysed. Each timber beam was defined according to the fibre direction (along the beam length). Two configuration setups were retained (Fig. 4):

To avoid any influence related to the boundary conditions, a free condition was reproduced by supporting the beam elements with very soft rubber bands (Fig. 4a). To ensure a free-based condition, the natural frequency of the last rigid mode should be less than one-tenth of the natural frequency of the first flexible mode [9].

A cantilever beam configuration was then adopted, with specific attention being paid to the one-side fixed condition. Since a perfect clamping was physically impossible without spoiling the wood surface (and therefore locally modifying the beam properties), neoprene pads were used between the beam and the support. This induced a unknown linear stiffness $k_{s}$ of the pad which then had to be estimated.

The roving hammer technique was applied, by exciting the structure vertically on all the selected points with an instrumented impulse hammer. Three piezoelectric accelerometers were vertically placed on the beam surface. The influence of the mass of these added accelerometers on frequencies is negligible, since their total mass was less than $5 \%$ of the weight of the timber beam. The obtained result was a series of measured accelerations at point $i$ for each excitation reference at point $j$, establishing the correspond- 


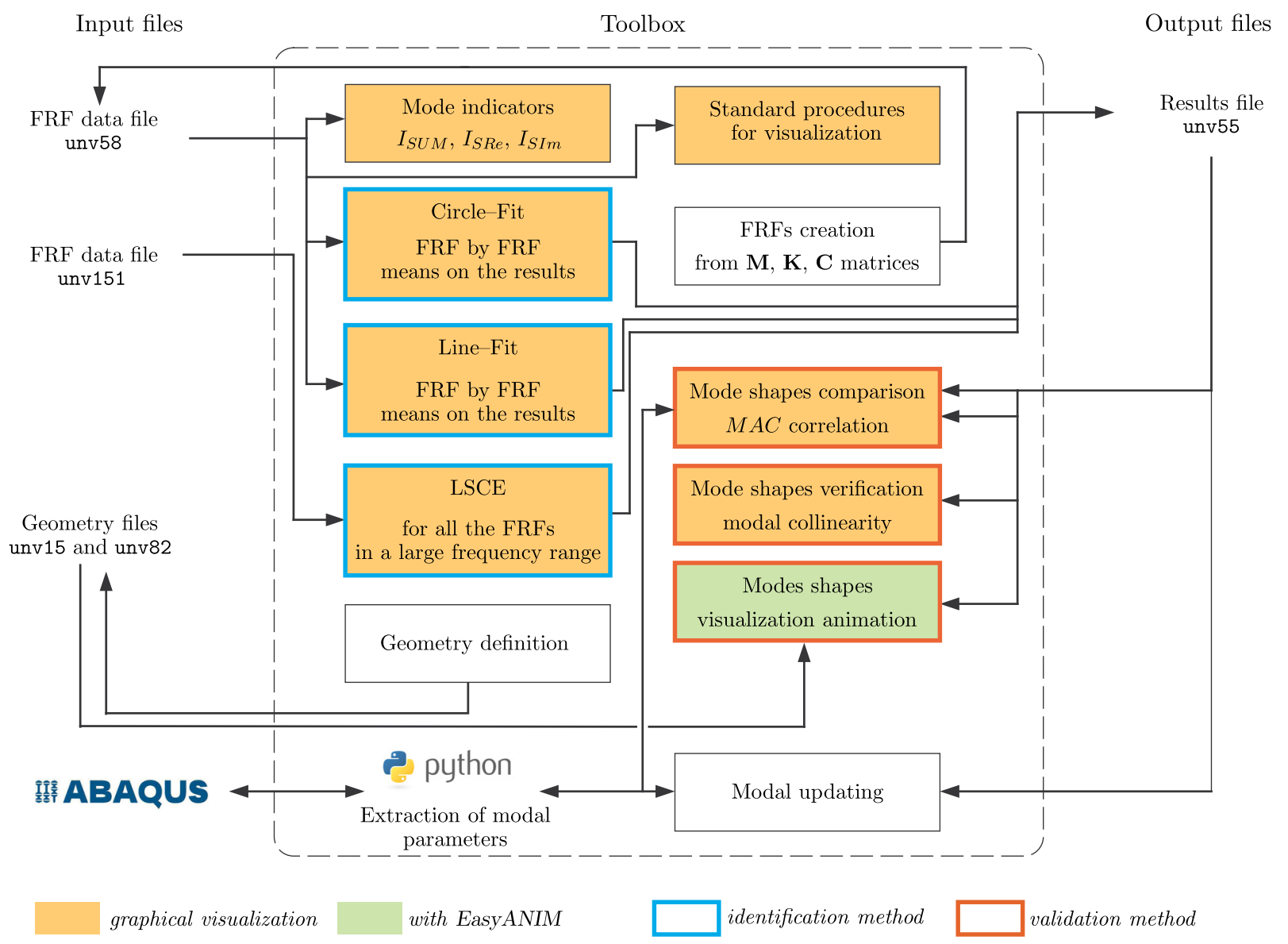

Fig. 5. Schematic operating diagram of the EasyMod toolbox, including the finite element updating procedure.

ing frequency response functions (FRFs) $H_{i j}(f)$ as a function of the frequency $f$. The applied frequency range was from $0-1024 \mathrm{~Hz}$ with a resolution of $1 \mathrm{~Hz}$. An exponential window was used to minimise the effects of leakage. According to the Betti-Maxwell reciprocity theorem, the excitation points $j$ and the response points $i$ can be switched $\left(H_{i j}(f)=H_{j i}(f)\right)$ so as to obtain a set of FRFs adapted to modal analysis. The least-square complex exponential (LSCE) method, implemented under the inhouse EasyMod toolbox [10,11], was used to determine the modal parameters (eigen frequencies, damping ratio, modal compliance) of the analysed structure.

\section{Implemented finite element updating procedure}

The EasyMod toolbox was used as it presents an alternative to commercial software packages for applying experimental modal analysis. It consists of several MatLab functions, allowing the step by step establishment of a complete modal analysis from experimental data. This tool has the advantage of being open source. The structure of EasyMod functions is presented in Figure 5. Both identification and validation methods are implemented: circle-fit, line-fit and LSCE methods, as well as modal assurance criterion $(M A C)$ and modal collinearity. By using the matrix abilities offered by MatLab, operations on FRF are made much easier.

The finite element analysis was performed using ABAQUS v.6.10 software. The model is a 3D beam element, defined in terms of bulk density $\rho$ and 9 mechanical constants: Young's moduli $E_{i}$, the shear moduli and Poisson's ratios $v_{i j}$. Indexes 1-3 correspond to the longitudinal, radial and tangential directions, respectively. Young's moduli and the Poisson's ratios are related by

$$
\frac{v_{i j}}{E_{i}}=\frac{v_{j i}}{E_{j}}, \quad i \neq j,
$$

to ensure the symmetry of the matrix. The direction of the axis of the anisotropic model was specified. In order to extract mode shapes, a set of nodes was defined, corresponding to the measurement setup. For the free boundary conditions, the hung rubber bands were modelled as springs whose stiffness $k_{b}$ was to be optimised during the modal updating. For fixed conditions, the pad stiffness $k_{s}$ was considered and included in the updating process. The goal of the procedure was to compare the modal parameters between the experimental measurements and the numerical simulations. To achieve the modal updating, the minimisation of one of several criteria (error functions) was considered [12]: 
- method 1: the frequency difference

$$
\varepsilon_{f, a}=\sum_{k}^{n}\left|f_{0, k}^{\exp }-f_{0, k}^{\text {num }}\right|,
$$

- method 2: the relative frequency difference

$$
\varepsilon_{f, r}=\sum_{k}^{n} \frac{\left|f_{0, k}^{e x p}-f_{0, k}^{n u m}\right|}{f_{0, k}^{e x p}}
$$

- method 3: $M A C$ criterion may be used under different forms to define an error function

$$
\varepsilon_{m a c}=\sum_{k}^{n}\left(1-M A C_{j k}\right) \quad \text { with } \quad j \neq k .
$$

An algorithm used to achieve the modal updating was implemented in EasyMod. It is based on a Python script created to extract the modal information (frequencies, damping ratios and mode shapes) from ABAQUS modal analysis results to be compared to ones of counterparts obtained from experiment. The updating is based on a minimum of unconstrained multivariable functions using a derivative-free method. The objective function was selected from the three aforementioned criteria using a large number of $n$ modes, so that the dynamic behaviour and the stiffness of the clamping or of the rubber bands could be estimated using an optimization algorithm [13].

With the intent to achieve modal updating, the mechanical behaviour of a timber beam relies on 10 parameters: $E_{1}, E_{2}, E_{3}, v_{12}, v_{23}, v_{13}, G_{12}, G_{13}, G_{23}$ and $\rho$, plus the boundary stiffness $\left(k_{s}\right.$ or $\left.k_{b}\right)$. If all of these parameters are considered for the modal updating, the number of variables is quite exhaustive and solving the problem would be badly conditioned. A numerical study was required to separate and study the influence of each parameter on the natural frequencies. Figure 6 shows the results from this sensitivity analysis, revealing that the effect of $E_{1}, G_{12}$ and $G_{13}$ on eigen frequencies $f_{0, i}$ is mainly positive although the Young's moduli $E_{2}$ and $E_{3}$ are insignificant. For instance, a relative variation of $200 \%$ of the Young's modulus $E_{2}$ only causes a change of $0.01 \%$ to the frequencies. With regards to the other mechanical parameters, they do not impact the frequencies. Obviously, since only bending modes are studied, Poisson's ratios do not impact the natural frequency results.

Another physical property of wood is the bulk density. This characteristic fluctuates severely according to the considered species, the position of the tree, and more precisely the position of the levied timber on the trunk. This is an important criterion which allows a physical description of wood. Moreover, the bulk density depends on the water content. Most commonly, the bulk density is considered for normal weather conditions (temperature of $20^{\circ} \mathrm{C}$ and relative air humidity of $65 \%$ ). This parameter should not be confused with the true density which corresponds to the ratio between the apparent density, and the porosity (practically identical whatever the considered species, $\left.1530 \mathrm{~kg} / \mathrm{m}^{3}\right)$.

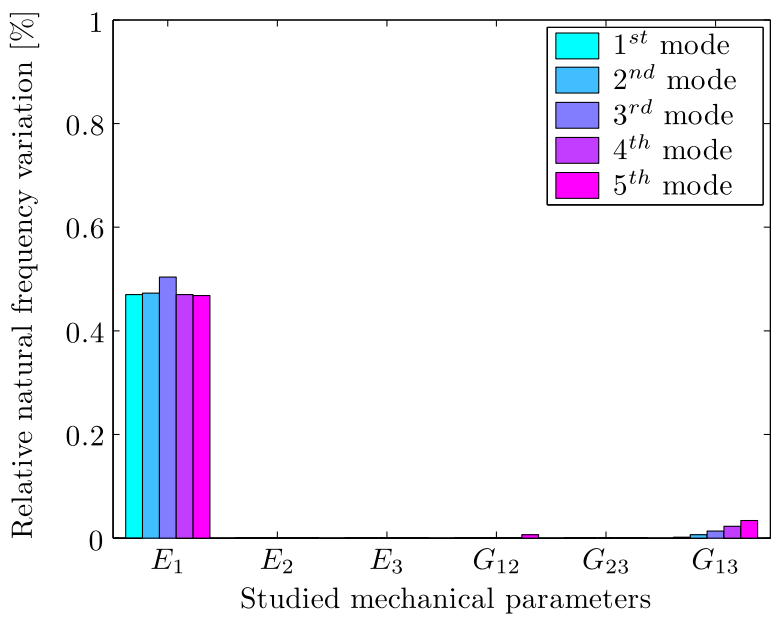

(a)

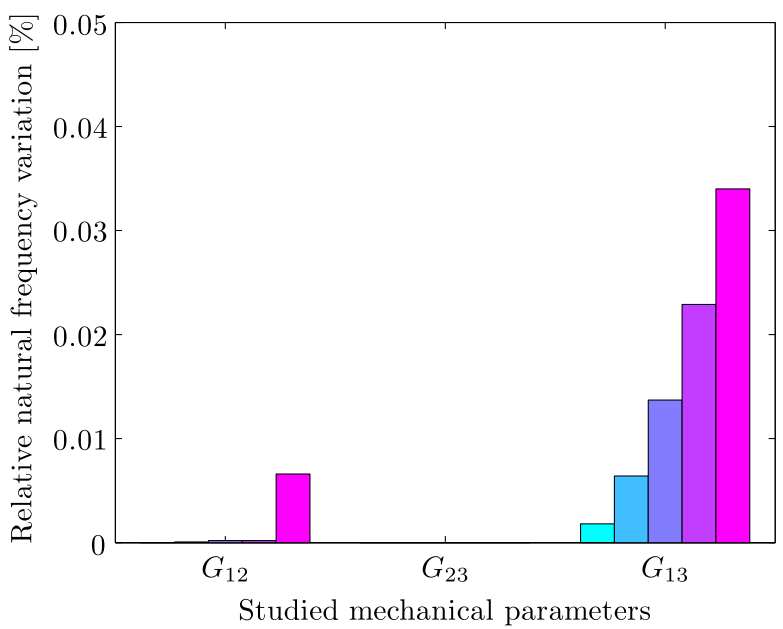

(b)

Fig. 6. Sensitivity study of the mechanical parameters: (a) all the moduli and (b) focusing on shear moduli.

\section{Results}

The timber beams were quite large $(14 \mathrm{~cm})$ and, due to the effects of knots or other heterogeneities, it was important to check if the side of the measurement, or its opposite side, had any influence on the natural frequencies. After several tests, the differences never exceeded more than $1 \%$, therefore the influence of the surface of measurement may be considered negligible. Other tests were performed, like the effect of local defects (holes or cracks) and it turns out that a small local modification on beam timber does not affect the modal properties.

Once the modal parameters were determined, several procedures that allowed the modal to be validated were used (visual verification, modal vector orthogonality,...). For instance, Figure 7 shows some FRF results obtained from impact measurements and the corresponding FRFs synthesised using the modal parameters extracted from the identification step. Clearly, both curves are accurately matched, serving as a check of the modal parameter estimation process. 


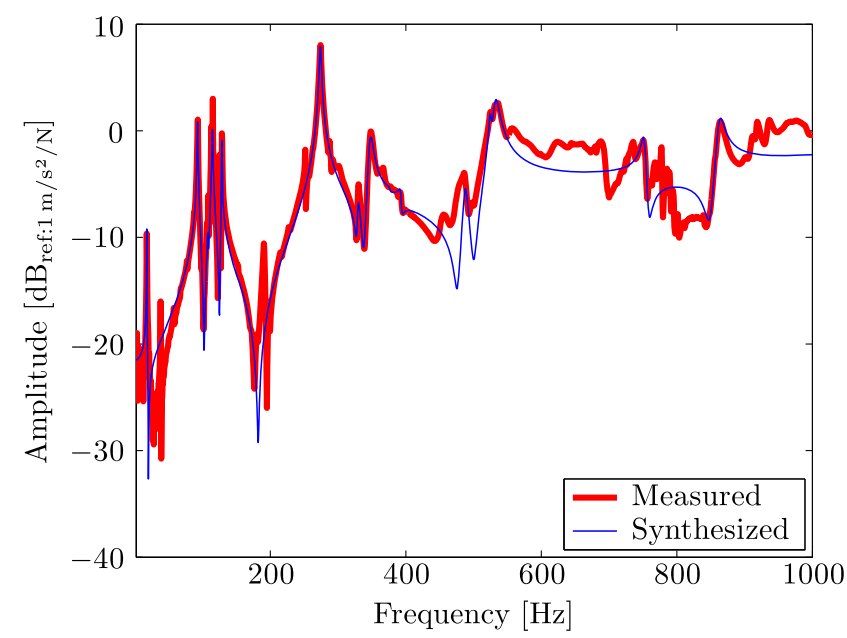

(a)

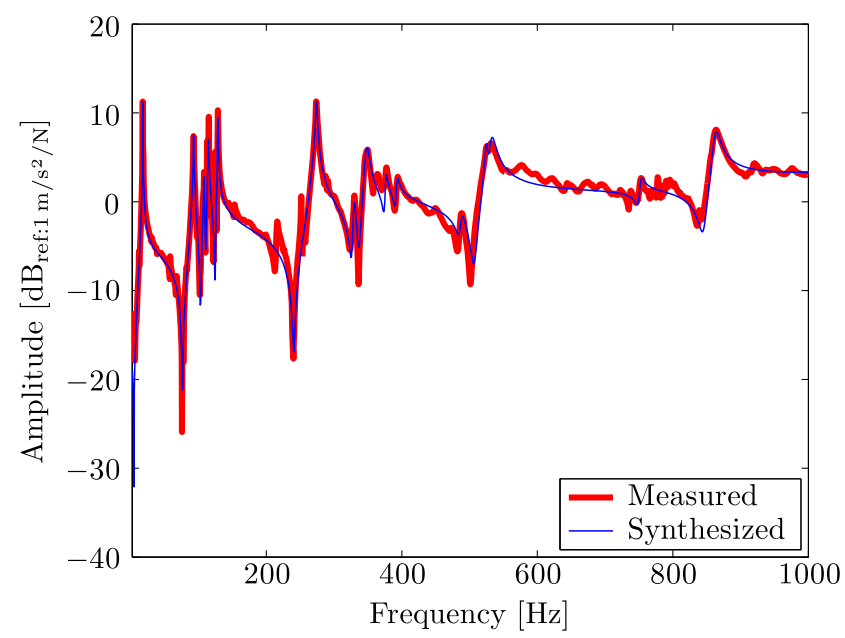

(b)

Fig. 7. Example of measured FRFs with the corresponding synthesised curve obtained from the estimated modal parameters.

The combination of the modal updating was tested for three aforementioned methods, defined by equations (2)(4). Each of them provided good results with low relative errors. The initial characteristics of the timber beams to initiate the updating was fixed to values found in literature: $\rho=475 \mathrm{~kg} / \mathrm{m}^{3}, \quad E_{1}=12000 \mathrm{MPa}, E_{2}=9067.4$ $\mathrm{MPa}, \quad E_{3}=332 \mathrm{MPa}, \quad v_{12}=0.33, \quad v_{13}=0.34, \quad v_{23}=0.48$, $G_{12}=600 \mathrm{MPa}, G_{13}=515.62 \mathrm{MPa}$ and $G_{23}=53.34 \mathrm{MPa}$.

With the aim of estimating the advantages and drawbacks of considering $E_{1}$ and $G_{12}$ or $E_{1}, G_{12}$ and $G_{13}$, the procedure was also carried out for each of these cases. Table 1 highlights the effectiveness of the procedure for the last mentioned case (simultaneously on the three parameters), showing the absolute and relative errors in natural frequencies and the $M A C$ values between the experimental and numerical mode shapes. It is shown that the proposed identification method allows an accurate identification of both in-plane and transverse elastic properties. Regarding the use of $M A C$ as a measure of consistency between estimates of a modal vector, it provides an efficient way of determining the degree of causality between the estimates of different modal vectors from the same system. It is simply defined, according to this approach, as follows:

$$
M A C_{i j}=\frac{\left(\left\{\psi_{i}\right\}^{T}\left\{\psi_{j}\right\} *\right)^{2}}{\left\|\psi_{i}\right\|^{2}\left\|\psi_{j}\right\|^{2}} .
$$

Although other forms of assurance criteria generally offer a better modal parameter estimation consistency [14], the form defined by equation (5) gives a reasonable definition to expected modal vectors and their accurate comparison, as illustrated in Figure 8 showing $M A C$ values of, almost always, greater than $90 \%$.

To verify the obtained dynamic parameter values, two other tests were used in parallel to the vibration tests: a visual inspection of the timber beam based on the Belgian national recommendation STS04 for estimating the mechanical characteristics STS04 [15] (qualitative estimation of $E_{S T S 04}$ based on a correspondence among various defined grating), and flexural tests carried out according to the standard NBN EN408 for the purpose of experimentally determining the Young's modulus $E_{1}$ [16]. This standard defines a set of conditions to be respected in order to carry out the test and to quantitatively estimate $E_{f l}$. The range $\left[E_{f l \text { min }}-E_{f l \text { max }}\right]$ defined by the last method was also provided and calculated by considering a tolerance of $0.5 \mathrm{~mm}$ on the measured deflection. Finally, the modal updating procedure was carried out for several timber beams and the results are presented in Table 2. At first sight, it seems that the results of the modal updating range from the values given by the STS04 guideline for the results of the flexural tests, except for some timbers (the bad results for beam $\# 2$ may be explained by the fact that during the flexural test, the timber had a clear trend to torsional buckling). With regards to these results, it appears that satisfactory results are provided by the modal updating, with a small difference between each method. This also pointed out the large discrepancies of the method provided by the standard NBN EN408. Both studied boundary conditions showed similar results and the assessment of the wood dynamic parameter with both cases was well deployed using the modal updating. In addition to the classical method, other parameters can be estimated, such as the damping loss factor measurable for each experimental test (a mean value around 0.003 was found through the different tests).

\section{Conclusion}

This paper analysed whether the procedure of modal updating ensures getting the mechanical characteristics of a timber beam using FRF measurements and finite element updating. This makes a link between the well-know method in mechanical engineering and the material used in civil engineering, for which the structural behaviour is still poorly understood. Based upon the results and findings obtained, the following conclusions were made:

- Free or fixed boundary conditions test can be used without any technical constraints and offer similar trends. 
Table 1. Example of results of the modal updating using method 3.

\begin{tabular}{ccclll}
\hline$f_{\text {exp }}[\mathrm{Hz}]$ & $f_{\text {num }}[\mathrm{Hz}]$ & $\Delta_{f}[\mathrm{~Hz}]$ & $\Delta_{f}[\%]$ & $M A C_{\text {before updating }}$ & $M A C_{\text {after updating }}$ \\
\hline 33.5 & 33.4 & 0.1 & 0.3 & 0.9977 & 0.9977 \\
91.2 & 91.2 & 0.0 & 0.0 & 0.9922 & 0.9992 \\
108.0 & 110.5 & 2.5 & 2.9 & 0.9631 & 0.9668 \\
177.3 & 176.1 & 1.2 & 0.7 & 0.9962 & 0.9962 \\
275.2 & 279.4 & -4.2 & 1.5 & 0.9146 & 0.9337 \\
281.7 & 285.3 & -3.6 & 1.3 & 0.9920 & 0.9923 \\
422.6 & 416.0 & 6.6 & 1.6 & 0.9885 & 0.9886 \\
494.9 & 491.8 & 3.1 & 0.6 & 0.8662 & 0.9163 \\
\hline
\end{tabular}

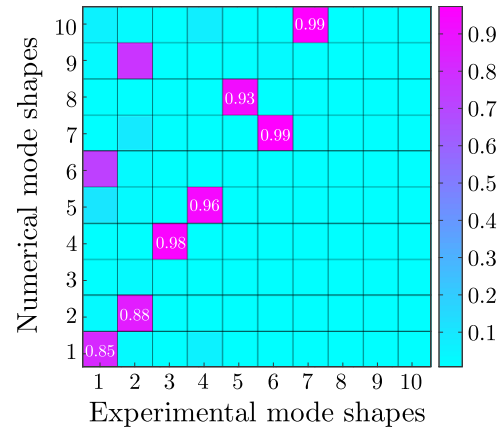

(a)

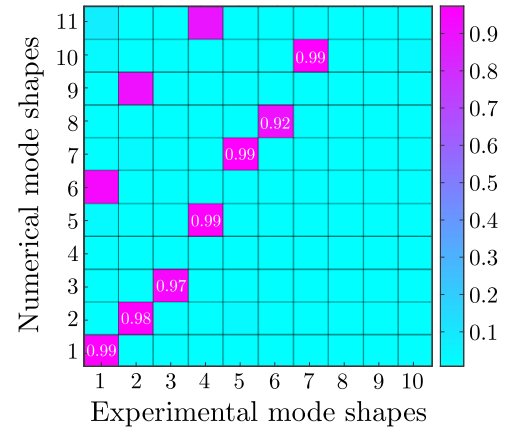

(b)

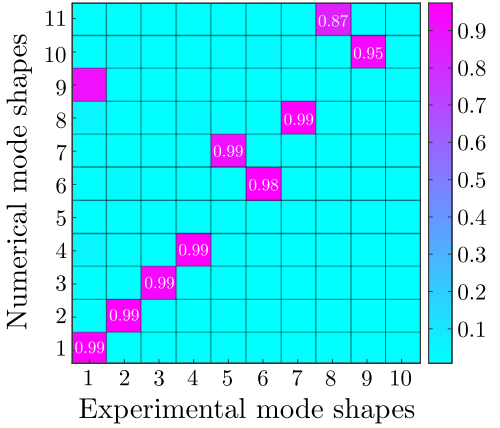

(c)

Fig. 8. Modal assurance criterion: (a) timber \#4, (b) timber \#5 and (c) timber \#14.

Table 2. Modal updating - Final results for some beam specimens (free boundary conditions).

\begin{tabular}{llllllll}
\hline \multirow{2}{*}{ Beam number } & $E_{S T S 0_{4}[\mathrm{MPa}]}$ & $\mathrm{E}_{f l}[\mathrm{MPa}]$ & $E_{f l, \min }[\mathrm{MPa}]$ & $E_{f l, \max }[\mathrm{MPa}]$ & & \multicolumn{2}{c}{ Young's modulus $E_{1}[\mathrm{MPa}]$} \\
\cline { 6 - 8 }$\# 1$ & $9000-10000$ & 13150 & 10270 & 18260 & 10650 & 10600 & 10500 \\
$\# 2$ & $9000-10000$ & 11970 & 8770 & 18850 & 5500 & 6000 & 5500 \\
$\# 3$ & $9000-10000$ & 13150 & 18260 & 10270 & 10500 & 10600 & 10500 \\
$\# 4$ & $9000-10000$ & 17110 & 13720 & 22740 & 13100 & 13200 & 13500 \\
$\# 5$ & $12000-13000$ & 16420 & 13340 & 21370 & 11900 & 12000 & 12000 \\
\hline
\end{tabular}

- Using beam elements allows the estimation of the longitudinal Young's modulus $E_{1}$ and, to a lesser extent, the corresponding shear moduli $G_{12}$ and $G_{13}$. The other dynamic parameters have no effect on the modal characteristics.

- Local defects, like holes or cracks, have a negligible influence on the natural frequencies so that the method could be generalised to use this technique on a large scale.

- Satisfactory results were obtained as the existing methods do not provide accurate results. This provides proof of the concept for the modal updating of timber beam using FRF measurement.

To allow the use of this assessment technique on site, the introduction of realistic boundary conditions in the process should be mastered.
Acknowledgement. The authors would like to acknowledge Amandine Meersseman and Pauline Goffinet for their various tests and analyses on multiple timber beams.

\section{References}

[1] B. Kasal, T. Tannert (Eds.), In situ assessment of structural timber, volume 7 of RILEM state of the art reports, Springer, Netherlands, 2011

[2] B.M. Lempriere, Ultrasound and elastic waves: frequently asked questions, Academic Press, San Diego, USA, 2003

[3] J.-L. Sandoz, B. Yann, Timber grading machine using multivariate parameters based on ultrasonic and density measurement, in: COST E 53 Conference - Quality Control for Wood and Wood Products, Warsaw, Poland, 2007, pp. $167-173$ 
[4] X. Yang, T. Amano, Y. Ishimaru, I. Iida, Application of modal analysis by transfer function to nondestructive testing of wood II: modulus of elasticity evaluation of sections of differing quality in a wooden beam by the curvature of the flexural vibration wave, J. Wood Sci. 49 (2003) 140-144

[5] Z. Wang, L. Li, M. Gong, Measurement of dynamic modulus of elasticity and damping ratio of wood-based composites using the cantilever beam vibration technique, Constr Build. Mater. 28 (2012) 831-834

[6] X. Wang, R.J. Ross, M.O. Hunt, J.R. Erickson, J.W. Forsman, Low frequency vibration approach for assessing performance of wood floor systems, Wood Fiber Sci. 37 (2005) 371-378

[7] R.J. Alves, M.D.C. Magalhaes, E.V.M. Carrasco, Determination of the transverse Young's modulus (TYM) of wood by means of an input power technique, Constr. Build. Mater. 42 (2013) 11-21

[8] A. Olsson, B. Källsner, Shear modulus of structural timber evaluated by means of dynamic excitation and fe analysis, Mater. Struct. 48 (2015) 977-985

[9] A.G. Piersol, T.L. Paez, Harris' shock and vibration handbook (6th Edition), McGraw-Hill, New York, USA, 2009
[10] G. Kouroussis, L. Ben Fekih, C. Conti, O. Verlinden, EasyMod: du développement d'un tool box sous MatLab vers l'enseignement des bases de l'analyse modale expérimentale, in: 3ième Colloque «Analyse vibratoire Expérimentale», Blois, France, 2012

[11] G. Kouroussis, L. Ben Fekih, C. Conti, O. Verlinden, EasyMod: a MatLab/SciLab toolbox for teaching modal analysis, in: 19th International Congress on Sound and Vibration (ICSV19), Vilnius, Lithuania, 2012

[12] J. Cugnoni, T. Gmür, A. Schorderet, Inverse method based on modal analysis for characterizing the constitutive properties of thick composite plates, Comput. Struct. 85 (2007) 1310-1320

[13] G. Kouroussis, L. Ben Fekih, T. Descamps, Assessment of timber element mechanical properties using experimental modal analysis, Constr. Build. Mater. 134 (2017) 254-261

[14] R.J. Allemang, The modal assurance criterion - twenty years of use and abuse, Sound Vib. 37 (2003) 14-21

[15] Service Public fédéral Economie, STS 04: Bois et panneaux à base de bois, Brussels, Belgium, 2008

[16] Institut belge de normalisation, NBN EN408: structures en bois - Bois de structure et bois lamellé collé - Détermination de certaines propriétés physiques et mécaniques, 2003

Cite this article as: G. Kouroussis, L.B. Fekih, T. Descamps, Using experimental modal analysis to assess the behaviour of timber elements, Mechanics \& Industry 18, 804 (2017) 